\title{
Left ventricle primary cardiac fibroma in an adult: A case report
}

\author{
XING-JU ZHENG and BIN SONG \\ Department of Radiology, West China Hospital of Sichuan University, Chengdu, Sichuan 610041, P.R. China
}

Received February 17, 2016; Accepted September 9, 2017

DOI: $10.3892 / \mathrm{ol} .2018 .9260$

\begin{abstract}
There is a very low prevalence of cardiac fibroma in the adult population. Cardiac fibromas arise from heart fibroblasts, and these tumors are primarily located in the ventricles or in the interventricular septum. Symptomatic tumors are treated by resection. By contrast, asymptomatic tumors require a long-term follow-up or surgical resection as a preventive measure to avoid complications. The present study reports the case of a 43-year-old man, who presented with a cough and shortness of breath for 2 months. Echocardiogram and cardiac magnetic resonance imaging indicated a large mass located in the left ventricular lateral wall. The patient underwent surgical excision of the tumor, and histopathological examination confirmed the mass to be a fibroma. The patient had a good postoperative recovery and was discharged on day 9 post-surgery.
\end{abstract}

\section{Introduction}

Cardiac fibroma is a rare benign primary tumor of the heart, which has been reported as the second most common benign cardiac tumor following rhabdomyoma in the pediatric population (1). Fibroma accounts for between 12 and $16 \%$ of primary cardiac tumors in children (2). Signs and symptoms are nonspecific, including arrhythmias, dyspnea, cyanosis, chest-pain and sudden mortality (3). However, a number of patients with cardiac fibroma are asymptomatic (4). The prevalence of cardiac fibroma is rare in the adult population (5). Cardiac fibromas generally are surgically resected, and patients with large tumors that cause heart failure require heart transplantation (2). Asymptomatic tumors require a long-term follow-up or surgical resection as a preventive measure to avoid complications (6). Echocardiography is the initial diagnostic modality for evaluating cardiac fibroma, and computed tomography (CT) or magnetic resonance imaging (MRI) can be as supplementary diagnostic techniques. The present case report describes an adult with cardiac fibroma arising from the left ventricle.

Correspondence to: Dr Bin Song, Department of Radiology, West China Hospital of Sichuan University, 37 Guoxue Xiang, Chengdu, Sichuan 610041, P.R. China

E-mail: binsong65@yahoo.com

Key words: fibroma, cardiac tumor, primary benign tumor, left ventricle, interventricular septum

\section{Case report}

A 43-year-old man presented with cough and shortness of breath, which continued for 2 months. The individual was admitted to the First People's Hospital of Chengdu (Chengdu, China) in June 2015, and a presumptive diagnosis of bronchitis was reached based on the aforementioned symptoms. Informed consent was obtained from the patient for publication of the present case report. Following relevant, standard treatment for bronchitis (chemotherapeutics and intravenous infusion therapy), the cough symptoms improved, however, the shortness of breath remained, and the patient complained of a slight chest pain. These symptoms had no association with regular activities such as walking. Electrocardiogram indicated normal sinus rhythm, and physical examination revealed normal blood pressure and regular pulse. Subsequently, echocardiography was performed, and revealed a large mass located in the left ventricular lateral wall. The patient was transferred to the West China Hospital, Sichuan University (Chengdu, China) for further investigation. Cardiac magnetic resonance imaging (MRI) indicated a large mass located in the lateral wall of the left ventricle. The mass was regular and well defined, with a size of 10x7 $7 \mathrm{~cm}$. Fig. 1A-D are MRI images captured of the $\mathrm{T} 1$ transaxial section, T2 transaxial section, T2 coronal section and T2 sagittal section, respectively. The mass was T1 iso-intense compared with cardiac muscle (Fig. 1A) and T2 hypo-intense compared with cardiac muscle (Fig. 1B-D). Myocardial first-pass perfusion images indicated a lack of enhancement in the mass compared to the myocardial wall (Fig. 1E). By contrast, in delayed phase perfusion images, the mass showed heterogeneous enhancement (Fig. 1F). In addition, MRI functional analysis reported no abnormality in cardiac function.

The patient underwent surgical excision of the tumor under standard cardiopulmonary bypass, and median sternotomy was performed. Under cardioplegic arrest and aortic cross clamping, the left ventricle was opened through the posterior wall. The tumor mass was fully resected, including a part of the lateral wall. The resultant defect was reinforced with a polytetrafluoroethylene patch. Histopathological examination conducted by the Pathology Department of West China Hospital, Sichuan University confirmed the mass to be a fibroma. The patient had a good postoperative recovery and was discharged on day 9 post-surgery. Follow-up echocardiography was performed every 3 months post-surgery, there is no evidence of recurrence and the patient remains asymptomatic. 

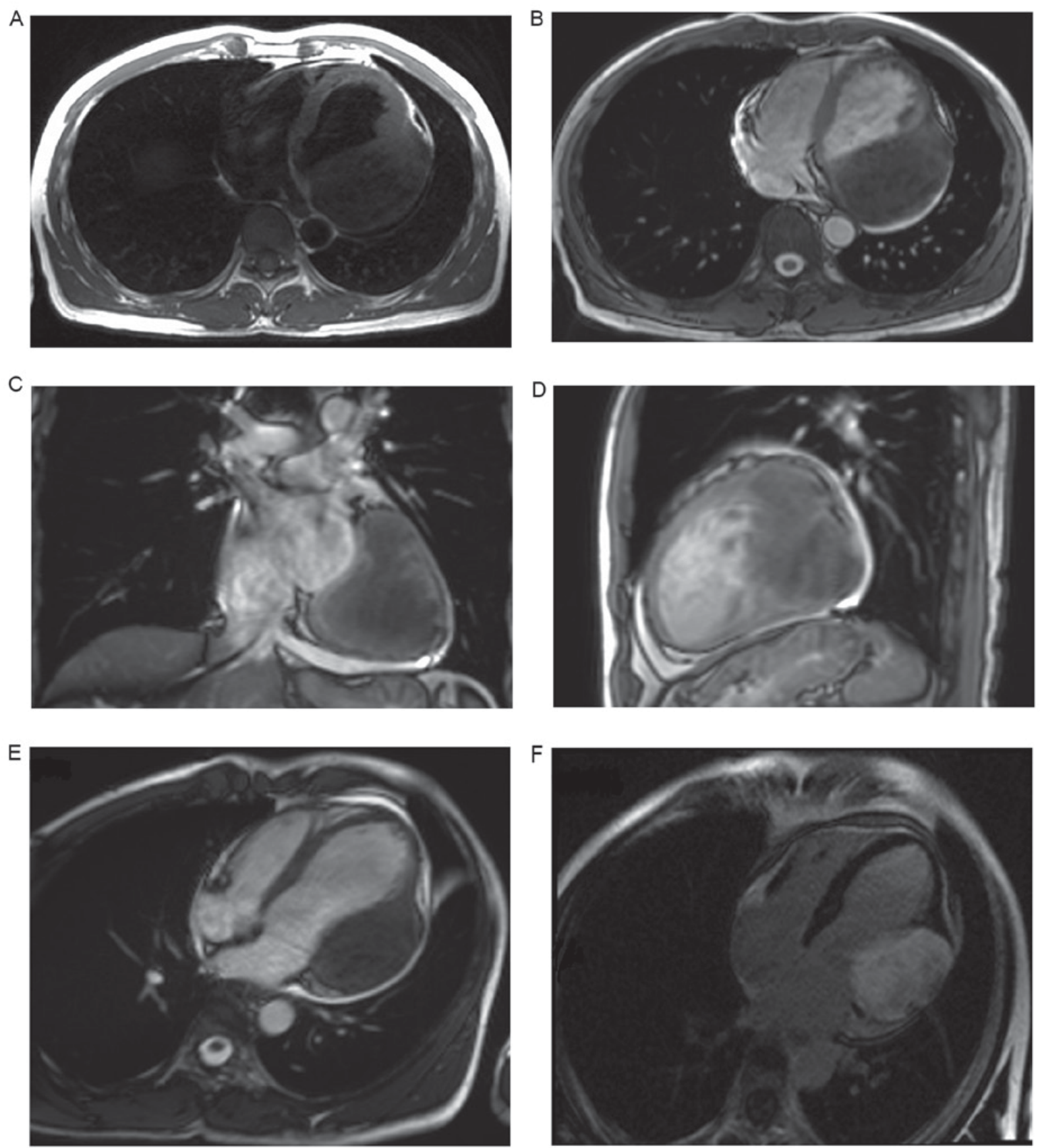

Figure 1. Cardiac fibroma in a 43-year-old male patient. Magnetic resonance imaging indicated a large mass located in the lateral wall of the left ventricle of the heart. (A) T1 transaxial section. (B) T2 transaxial section. (C) T2 coronal section. (D) T2 sagittal section. The mass was T1 iso-intense and T2 hypo-intense relative to muscle. (E) Myocardial first-pass perfusion image indicated no enhancement in the tumor. (F) Delayed-phase perfusion image showed heterogeneous enhancement in the tumor.

\section{Discussion}

Cardiac tumors may be primary or secondary tumors (4). Primary cardiac tumors are rare, with a prevalence of $<0.03 \%$ according to postmortem studies (1). Cardiac fibromas arise from heart fibroblasts, and these tumors are mainly located in the ventricles or interventricular septum (4). Cardiac fibromas generally have no capsules. Fibromas are able to interdigitate with ventricular muscle at the tumor border and replace functioning muscle mass, which may result in intractable congestive heart failure $(2,6)$. Clinical symptoms and signs of cardiac tumors vary depending on tumor location and size (7). Cardiac tumors may be associated with certain symptoms, including chest pain, cardiomegaly, arrhythmias and even sudden mortality. In some cases, cardiac tumors may be asymptomatic $(1,8)$.

Echocardiography is non-invasive, fast and does not involve the use of radiation. It is generally the initial diagnostic modality for evaluating cardiac fibroma. Supplementary diagnostic techniques include computed tomography (CT) or MRI. CT and MRI can provide the location of the tumor, as well as identifying its surrounding structures and hemodynamic effects. In addition, MRI can provide additional functional data. Therefore, cardiac MRI is the modality of choice for further evaluation of cardiac fibroma $(1,4)$. 
In the present study, the cardiac MRI showed a regular solid lesion, which was iso-intense relative to muscle on T1-weighted and hypo-intense on T2-weighted images, which suggested fibrosis (9). Diagnosis was confirmed by histopathological evaluation of the specimen following surgical resection.

Cardiac tumors, that are symptomatic, are treated by surgical resection (3), and if surgical resection is not possible, heart transplantation is required (1). The type of surgical intervention (a total or subtotal resection) depends on the location of the tumor (1) with total and subtotal resection reported exhibit good outcomes (10). Asymptomatic tumors require a long-term follow-up or surgical resection as a preventive measure to avoid complications (6). In the present study, the patient presented with symptoms, and therefore surgery was required. Following operation, the patient had a good recovery, and echocardiography, which was performed 3 months following surgical resection, indicated no recurrence.

In conclusion, cardiac fibroma is very rare in adults. Echocardiogram, CT and cardiac MRI can provide valuable findings. Surgical excision is a reliable and effective method for treatment.

\section{References}

1. Ünsal H and Ekici E: Conservative management of a left ventricle cardiac fibroma in an asymptomatic child patient. Turk Kardiyol Dern Ars 43: 481-483, 2015.

2. Uzun O, Wilson DG, Vujanic GM, Parsons JM and De Giovanni JV: Cardiac tumours in children. Orphanet J Rare Dis 2: 11, 2007.

3. Burke A and Tavora F: The 2015 WHO classification of tumors of the heart and pericardium. J Thorac Oncol 11: 441-452, 2016.

4. Tao TY, Yahyavi-Firouz-Abadi N, Singh GK and Bhalla S: Pediatric cardiac tumors: Clinical and imaging features. Radiographics 34: 1031-1046, 2014.

5. Cho SH, Fritz T, Cronin LJ and Cohle SD: Primary cardiac fibroma in an adult. Case Rep Cardiol 2015: 713702, 2015.

6. StillerB,HetzerR,Meyer R,Dittrich S,Pees C,Alexi-Meskishvili V and Lange PE: Primary cardiac tumours: When is surgery necessary? Eur J Cardiothorac Surg 20: 1002-1006, 2001.

7. Bruce CJ: Cardiac tumours: Diagnosis and management. Heart 97: 151-160, 2011.

8. Burke AP, Rosado-de-Christenson M, Temleton PA and Virmani R: Cardiac fibroma: Clinicopathologic correlates and surgical treatment. J Thorac Cardiovasc Surg 108: 862-870, 1994.

9. Eric S, Ana S and Philippe D: Inter-ventricular septal cardiac fibroma in an adult: MR and MDCT features with pathologic correlation. Eur J Radiol Ext 67: e103-e106, 2008.

10. Cho JM, Danielson GK, Puga FJ, Dearani JA, McGregor CG, Tazelaar HD and Hagler DJ: Surgical resection of ventricular cardiac fibromas: Early and late results. Ann Thorac Surg 76: 1929-1934, 2003. 